DOE/ER/60791--1

DE93 003085

\title{
INVESTIGATION OF DISPERSION IN POROUS MEDIA USING FIBER-OPTIC TECHNOLOGY
}

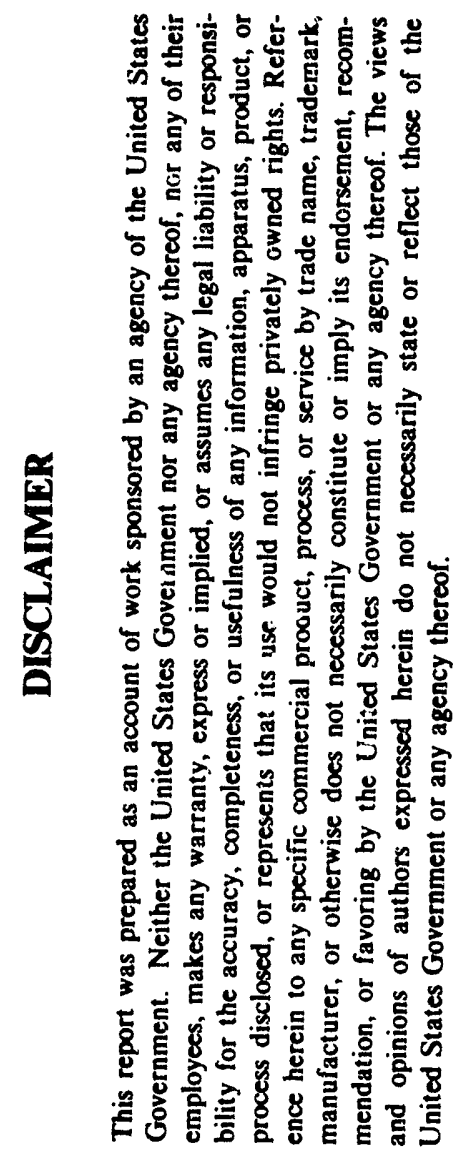

\author{
J. Mark Nielsen \\ ENVIRON Corporation \\ Princeton, New Jersey \\ George F. Pinder \\ College of Engineering and Mathematics \\ University of Vermont \\ Burlington, Vermont \\ Thomas J. Kulp \\ Lawrence Livermore National Laboratories \\ Livermore, California \\ Stanley M. Angel \\ Lawrence Livermore National Laboratories \\ Livermore, California
}


This paper presents the results of a laboratory investigation of the characteristics of solute transport utilizing fiber-optic technology. In this work, we examine solute transport in a uniform homogeneous porous medium with a one dimensional flow system using a fiber-optic fluorimeter system. In a series of experiments, fiber-optic sensors are used in-situ in a packed column to make observations of species concentration at the microscopic level. The tracer that is employed is assumed to be ideal and the flow field is restricted to be fully saturated and laminar. Using data measured at the pore level, we study the spatial variability in the concentration ield during miscible displacement and relate these observations to the macroscopic flow characteristics. 


\section{INTRODUCTION}

While considerable effor has been expended in the study of dispersion in porous media using macroscopic level observations of species concentrations, relatively little work has utilized observations taken at the microscopic level. In this paper we present a series of experiments that employ observations taken at the microscopic level, using fiber-optic technology, to examine dispersion in a packed column.

Earlier efforts to determine the microscopic behavior of fluids in porous media have taken two approaches. Dybbs and Edwards (1986) used laser doppler anemometry to study interstitial velocities. They measured microscopic flow velocity characteristics that had previously only been inferred from mactoscopic observations. The first use of fiber-optic technology in the study of flow in packed columns was performed by Kulp et al. (1987). They demonstrated that fluid contamination can be monitored at several points in a column packed with glass beads using fiber-optic probes. These researchers did not, however, evaluate their results in terms of dispersion.

In our work we ernployed the techniques of Kulp et al. (1987) to examine solute transport in a uniform homogeneous porous medium with a one dimensional flow system. Using data measured at the pore level via fiber-optic technology, we study the spatial variability in the contamination field from point to point within the porous media and relate the observations to the macroscopic flow characteristics. The tracer that is employed is assumed to be ideal and the fully saturated flow field is restricted to the laminar range.

\section{EXPERIMENTAL PROCEDURES}

The experimental apparatus (see Figure 1) is designed to measure the change in concentration of a fluorescent dye dissolved in a flowing fluid at several pore locations within a column packed with glass beads. The packed column consists of a clear lucite 
tube approximately one meter in length with an inside diameter of approximately $10 \mathrm{~cm}$. The length of the column was selected to reduce apparatus induced dispersion since this effect is most significant in short columns (.James and Rubin, 1972). The inside diameter was chosen to reduce wal! effects to a level consistent with the objectives of the experiment. Wall effects have been investigated by Schwartz and Smith (1953), Lyczkowski (1982), and Dybbs and Edwands (1986). Their investigations indicate that unless the column to bead diameter ratio is greater than 30 , there will be significant variations in flow velocities across the column. Thus the column diameter was scaled to the bead size.

A bead size of $3.0 \mathrm{~mm}$ was selected to minimize the influence of the 200 micron core diameter fiber probes that were used to measure the fluorescent tracer concentration in the pores. Having selected this bead size, a column diameter of $10.16 \mathrm{~cm}(4 \mathrm{in}$.) was chosen to produce a column diameter to particle ratio of 33.68 , which is greater than the ratio of 30 recommended by earlier workers to provide for minimal wall effects.

To further assure that wall effects did not unduly influence the experiment, the pores to be monitored for concentration change were selected so as to be a minimum distance from the column wall. Studies on wall effects indicate that the maximum pore velocity $\propto c c u r s$ at a distance of approximately one particle diameter from the wall, regardless of the ratio of column to particle diameter. These observations were confirmed by the results of Dybbs and Edwards (1986) who also observed that the velocity reaches its minimum value at approximately 1.5 particle diameters from the wall. This leads to the conclusion that as long as the fiber-optic probes lie at least $6.0 \mathrm{~mm}$ (two bead diameters) from the column wall, the concentration measurements will not be unduly influenced by the wall effects.

The one dimensional flow system was driven by a constant head tank (see Figure 1) mounted above the column. The constant head tank is supplied by two separate supply 
reservoirs, one containing water and the other containing a solution of Uranine, a purified grade of Fluorescein sodium salt, dissolved in water. Constant head is maintained in the head tank using an overflow weir and the flow rate is determined by directing outflow from the column to a volumetric flask and measuring the amount of fluid collected per minute.

The tracer concentration measuring system consists of a light source, an optical fiber, a fluorimeter and an automated data collection system. The increase in fluorescence encountered during the breakthrough of the displacing tracer was measured using a dedicated remote fiber fluorimeter system. This system delivers visible light to the sampling region, in this case the pore space, via an optical fiber and then collects the fluorescent light emitted by the tracer dye using the same fiber. To assure that the exact location of the fiber tip was known, each tip was located in an artificial pore created from eight beads arranged in cubic packing. This artificial prre with the embedded fiber was, in urm, placed in the column. The use of a single fiber to emit and receive light is important since it reduces the required probe size. The smaller probe size reduces the disruption of the flow field by the fiber. Because the fluorimeter system developed by Kulp et al. (1987) was designed to collect fluorescent emissions from a single optical fiber, while the intent of our experiment was to monitor several locations in the column simultaneously, a fiber-optic multiplexer system was employed to enable the use of eight fiber-optic probes.

The fiber-optic system was calibrated to compensate for signal losses. Such losses can be due to the effective path length of each fiber and the fiber connectors. In addition losses may be expected from the multiplexer connectors. A series of preliminary experiments were conducted to determine the functional form of the response of the fluorimeter system to tracer concentrations at each fiber tip (Nielsen, 1988). The resulting curves were then used in the interpretation of the fiber-optic data. 
The experimental design ciescribed in the next section requires a large number of individual experimental runs. One experimental run consisted of measuring the concentration profile for a complete miscible displacement of the initial resident solution $(C=0)$ by a tracer solution $(C=C 0)$. Measurements were made at eight locations arranged in a radial pattem in a plane perpendicular to the average flow direction (see Figure 2). Four probes were inserted so that their tips were $1.9 \mathrm{~cm}(0.75 \mathrm{in})$ from the column wall and the tips of four more were located $3.8 \mathrm{~cm}(1.5 \mathrm{in})$ from the column wall.

\section{EXPERIMENTAL DESIGN}

A factorial experimental design was used to examine the influence of three factors on the amount of dispersion observed in the column. The factors evaluated were the macroscopic fluid velocity, the distance of the observation point from the entry point of the tracer, and the relative time. The volumetric flow rates that were considered were $30 \pm 1$, $15 \pm 1$, and $5 \pm 1 \mathrm{ml} / \mathrm{min}$. The measurement locations along the column were at 27.3 and $78.1 \mathrm{~cm}$ from the inlet. The relative time is determined by examination of the relative concentration breakthrough curve. For each run the time at which the relative concentration, as determined from theoretical curves to be discussed later, reached 0.25 , 0.5 and 0.75 was noted. These times are hereinafter denoted as the relative times.

In order to enhance the probability that the experiment would provide statistically significant results, five experimental runs were made for each factorial combination. The factorial experiment is summarized in Table 1. 
Table 1: Experimental Run Information

\begin{tabular}{lccc}
\hline $\begin{array}{l}\text { Experiment } \\
\text { Set No. }\end{array}$ & $\begin{array}{c}\text { Measurement } \\
\text { Location } \\
(\mathrm{cm})\end{array}$ & $\begin{array}{c}\text { Flow Rate } \\
(\mathrm{m} / \mathrm{min})\end{array}$ & $\begin{array}{c}\text { Number of } \\
\text { Reperitions }\end{array}$ \\
\hline & 78.1 & $30 \pm 1$ & 5 \\
1 & 78.1 & $15 \pm 1$ & 5 \\
3 & 78.1 & $5 \pm 1$ & 5 \\
4 & 27.3 & $30 \pm 1$ & 5 \\
5 & 27.3 & $15 \pm 1$ & 5 \\
6 & 27.3 & $5 \pm 1$ & 5 \\
\hline
\end{tabular}

Consider for a moment the information presented in Table 1. The experiments are set up so that the level of every factor appears in combination with the level of every other factor. The relative time factor does not appear in this table because the concentration measuring equipment generates a continuous in time curve from which the desired time values can be selected. The table indicates that six experiments must be conducted, each repeated five times. This yields a total of 30 experiments. Because the relative time was evaluated at three levels, this experiment can be described as a $2 \times 3 \times 3$ factorial design.

Inasmuch as only eight fibers were available for making measurements, two fiber arrangements had to be considered. Consistent with our earlier comments regarding measurement locations, each fiber arrangement consisted of four probes positioned so that their tips were $1.9 \mathrm{~cm}(0.75 \mathrm{in})$ radially from the column wall and four others so that their tips were $3.8 \mathrm{~cm}$ ( $1.5 \mathrm{in}$ ) from the column wall. For the first three set of experiments eight fibers were located at a distance of $78.1 \mathrm{~cm}$ (30.7 in) from the top of the packed section of the column; for the second three set of experiments the fibers were located at $27.3 \mathrm{~cm}$ (10.7 in) from the top. Using this strategy, only eight fibers were ever required for a given 
experiment. Note also that by positioning the fibers at two radial distances from the wall, it is possible to examine the distance from the wall as a separate factor. The influence of this factor, however, was not evaluated.

Three design restrictions governed the decision to use the designed flow velocities. To assure that the velocities stayed within the laminar range so that Darcy's law would remain applicable, the flow rate was selecied so that the Reynolds number did not exceed unity. For a bead size of $3.0 \mathrm{~mm}$ a Reynolds number of unity yields a flow rate of $166 \mathrm{ml} / \mathrm{min}$. The lower bound on the flow rate was selected to assure that dispersive mixing dominated over molecular diffusion. Based upon published experimental data, a lower bound for the Peclet number of 100 was selected. This translated into a lower bound for the flow velocity of $2.8 \mathrm{ml} / \mathrm{min}$.

\section{EXPERIMENTAL RESULTS}

To show that the variable concentration fields do exist at the pore level, plots of the concentration behavior at three specified times are presented in Figure 3. These plots show a perspective view of the concentration field. They represent the concentration distribution over a cross-section of the column perpendicular to the direction of average fluid velocity. The vertical axis represents the normalized concentration. The individual peaks, most clearly apparent in the later in time plots, represent the normalized concentration at the fiber-optic tip locations seen earlier in Figure 2.

It is evident from this figure that the concentration at the various pore locations begins to increase at some pores earlier than others. At a time of 0.5 , the point 2: which the average normalized concentration over the cross-section is about 0.5 , the difference between the various concentration values is quite pronounced. By a time of 0.75 , the concentration values are approaching one another because the concentration at certain of the 
fibers is beginning to asymptotically approach unity.

One should note that in the perspective plots for all of the experiments, none consistently showed the data spikes closer to the column wall growing more rapidly that those further from the wall. This uggests that wall effects did not play an important role in the experiments.

Another plot of this behavior is represented in Figure 4. The concentration at each fiber tip is presented as a function of time, where time is represented by the volume of injected tracer. The difference in the concentration profiles appears to be due primarily to a shift in the curves, rather than a significant change in their shape. The solid curve represents an Ogata (1970) solution to the transport equation with the dispersion coefficient selected based on the computed Peclet number and using empirical relationships given by Bear (1979). The dispersion coefficients calculated using these empirical relationships translated into an approximate dispersivity of $0.5 \mathrm{~cm}$. In most cases, the observed data deviate equally on either side of the computed profile when compared to the shifted analytical solution. This behavior indicates a variation in concentration about an average represented by the theoretical solution.

To determine the dependence of the concentration variability on the flow rate, time, and travel distance (or position of measurement) an analysis of variance was performed on the results from the factorial design. The first step in the analysis is the definition of a measure of spatial variability in concentration. We selected two measures. One is the variance in the measured concentration field at a specific point in time. Thus, denoting spatial variability by SP, we have:

$$
S P=\frac{1}{n-1} \sum_{i=1}^{n}\left(C_{i}-\bar{C}\right)^{2} ; n=8
$$

where $C$ is the average concentration over the porous media cross-section. As indicated 
earlier, the concentrations appearing in equation 1 have been normalized with respect to the input concentration $\mathrm{C} 0$.

An ana'ysis of variance associated with the factorial experiment described earlier was used to establish whether or not the factors considered in the experiment had a significant impact on the concentration variability described by the parameter SP. The analysis of variance indicated that the effects of flow rate, relative time, position, and the interaction between flow rate and position on the concentration variability were significant at the 95 percent confidence level.

The factorial design also allowed the determination of the polynomial relationship between the various factors and the concentration variability. Because only two positions in the column were examined, it was possible to consider only a linear variation in this factor. Using three different flow rates and three different relative times, it was possible to consider only a quadratic variation in these two factors. We now consider each of these cases. In the following discussion, all statistical tesis are evaluated such that significance implies statistical significance at the 95 percent confidence level.

Figure 5 represents the relationship between the spatial variability SP and the position of the observation point relative to the top of the packed column. Because there are three factors involved in the experiment, the results of 90 experiments are presented but the specific replicate values are not shown. Thus each plot in Figure 5 has recorded the linear relationships associated with a particular relative time. In addition, there are three curves on each plot, each curve indicating the behavior associated with a particular flow rate. Analysis or variance indicates that, between the two measurement locations in the column, there was a significant overall decrease in the amount of concentration variability as one moves further from the source of the tracer. It is also evident, as indicated by the analysis of variance, that the behavior observed between the two mcasuring points depends upon 
the flow rate considered. For example, at the highest flow rate there is a significant increase in the spatial variability of the contamination as one moves down the column from the source of the tracer. In retrospect, it would have been interesting to have had at third sampling location along the column to establish whether or not there was a quadratic variation in concentration variability versus distance from the source. Unfortunately these measurements could not be made due to column construction.

Figure 6 illustrates the relationship between the spatial variability of the concentration and the flow rate. Because three flow velocities were considered, a quadratic relationship could be tested. The fact that a significant quadratic relationship was observed over the range of flow rates considered is clearly evident in this figure. The three plots represent once again observations made at different relative times. The two curves on each plot indicate behavior at the two locations along the column. It is evident from these curves that the spatial variability of the concentration is smaller at a flow rate of $15 \mathrm{ml} / \mathrm{min}$ than at lower and higher flow rates.

Figure 7 indicates the relationship between the spatial variability in concentration and the relative time. Because three relative time values were selected, a quadratic relationship was tested and found to be significant. The linear term, however, was not significant. As the curves illustrate, the spatial variability is highest when observations are made at approximately the midpoint of the breakthrough curve. It is interesting to note that there was no significant correlation between the relative time and flow rate or position.

While the results presented above are given for the case when the spatial variability is defined as the variance in the concentration, as mentioned earlier, an alternative measure of variability was also considered. Thus, defining spatial variability as MX, we have: 


$$
\mathrm{MX}=\operatorname{maximum} \text { of }\left(\mathrm{C}_{\mathrm{i}}-\mathrm{C}_{\mathrm{j}}\right) ; \mathrm{i}, \mathrm{j} \varepsilon \mathrm{I}
$$

where $I$ is the number of observations in a section. The experimental results analyzed using this measure showed the same relationships as indicated above.

In an effort to view the variability of the concentration in three space dimensions, a transformation was applied to the time dependent data. Assuming that, with respect to the fluid flow, space and time are related through the fluid velocity, and assuming the average pore velocity, as represented by the macioscopic velocity, is indicative of the true pore velocity, one can interchange spatial and temporal integrations. Thus by averaging the behavior of the concentration in the pore over boin the cross-section and the representative times, we have an approximate representation of contamination behavior in three space dimensions.

The results of the time averaging procedure are presented in Figure 8.

\section{DISCUSSION OF RFSULTS}

The above described experiment addresses the question of the relationship between the macroscopic measurement of concentration variability, commonly known as dispersion, and the notion of concentration variability as observed at the pore level. Heretofore, experiments conducted to examine the phenomenon of dispersion focused on macroscopic observations, usually taken at different times at the end of a porous media filled column. In our experiment we attempted to relate the microscopic and macroscopic worlds through the use of fiber-optic observations of tracer concentrations at the pore level. The resulting pore level observations were then examined for their variability. By selecting different flow rates, positions, and relative times of observation, a relationship between the pore level 
variability and these factors was established. These relationships are presented in Figures 5 through 7.

One can attempt to interpret the results of this experiment within the context of the theory of volume averaging. Fundamental to this coninuum mechanical concept is the idea of a representative elementary volume, such as described by Bachmat and Bear (1985). In such a volume of porous media it is possible to integrate the values of certain parameters so as to obtain smoothly behaved averaged quantities as one moves from point to point. In the case of the tracer used in this experiment, the averager quantity would be the concentration. Dispersion is associated with the deviation of the concentration and velocity tields from their spatially averaged behavior. One measure of dispersion, as distinct from the dispersive flux, is the concentration variance.

The cross-section wherein our observations were taken could be considered as a two-dimensional representative elementary volume. The variability in pore level concentrations from their mean value would, in such a model, constitute dispersion. Our experiment is designed to establish whether the dispersion described in the above manner has the same physical behavior as dispersion observed using measurements at the macroscopic level.

The two phenomena that have corresponding relationships in experiments conducted using observations at both the macroscopic and microscopic levels are the change in dispersion with distance traveled down a column from the source and the dependence of dispersion on macroscopic flow velocity. Our experiments indicate a decrease in dispersion with distance from the source. This is in conflict with experiments of others (see, for example, Blackwell, 1962). However, it must be kept in mind that our experiments were designed to reveal only linear changes with distance, and it may well be 
that a quadratic relationship exists that would have resulted in higher dispersion at greater distances.

The relationship between flow and dispersion is consistent with the experiments of others who used macroscopic observations. The dispersion decreases initially and then, at higher flow velocities, increases.

A relationship we are able to observe that has not been observed in macroscopic experiments is the dependence of dispersion on the relative breakthrough time. We found that dispersion early in the breakthrough curve increases with time, reaches a maximum at the point when the average relative concentration is approximately 0.5 and then decreases. This is consistent with our expectations, since early and late in the breakthes gh curve the relative concentrations are bounded below by zero and above by one.

In summary, the experiments presented above demonstrate that it is possible to move from the microscopic level of the pore to the macroscopic level of the porous medium using the concepts associated with volume averaging. It demonstrates, at least at the column level, the existence of a meaningful representative elementary volume. 


\section{REFERENCES}

Bachmat, Y., Bear, J. On the Concept and Size of a Representative Elementary Volume (REV). Advances in Transport Phenomena in Porous Media. Proceedings of the NATO Advanced Sudy Institute on Fundamentals of Transpor. Phenomena in Porous Media. Newark, Del., July 14-23, 1985.

Bear, J. Hydraulics of Groundwater. McGraw-Hill, Inc., New York, NY, 1979.

Blackwell, R. J. Laboratory Studies of Microscopic Dispersion Phenomena. Society of Petroleum Engineers Journal, v51, pp1-7, March 1962.

Dybbs, A., Edwards, R. V. A New Look at Porous Media Fluid Mechanics - Darcy to Turbulent. Laser Anemomeroy in Eluid Mechanics II. Selected Papers from the Intemational Symposium on Applications of Laser Anemometry to Eluid Mechanics. Ladoan, Lisbon, 1986.

James, R. V. and Rubin, J. Accounting for Apparatus Induced Dispersion in Analyses of Miscible Displacement Experiments. Water Resources Research, v8, no.3, pp717-721, June 1972.

Kulp, T. J., Bishop, D., and Angel, S. M. Column Profile Measurements Using Fiber-Optic Spectroscopy. Preprint paper, Lawrence Livermore National Laboratory, University of California, Livermore, CA. July 1987.

Lyczkowski, R. W. Modeling of Flow Nonuniformities in Fissured Porous Media. Canadian Joumal of Chemical Engineering, v60, pp61-75. February 1982.

Nielsen, J. M. A Study of the Spatial Variability of Microscopic Solute Transport in Dispersive Flows Using Fiber-Optic Sensors. M.S.E. Thesis, Princeton University. Princeton, NJ, 1988.

Ogata, A. Theory of Dispersion in a Granular Medium. U.S. Geological Survey Paper 411-I, pp134. 1970.

Schwartz, C. E. and Smith, J. M. Flow Distribution in Packed Beds. Industrial and Engineering Chemistry, v45, no.6, pp1209-1218. 1953. 

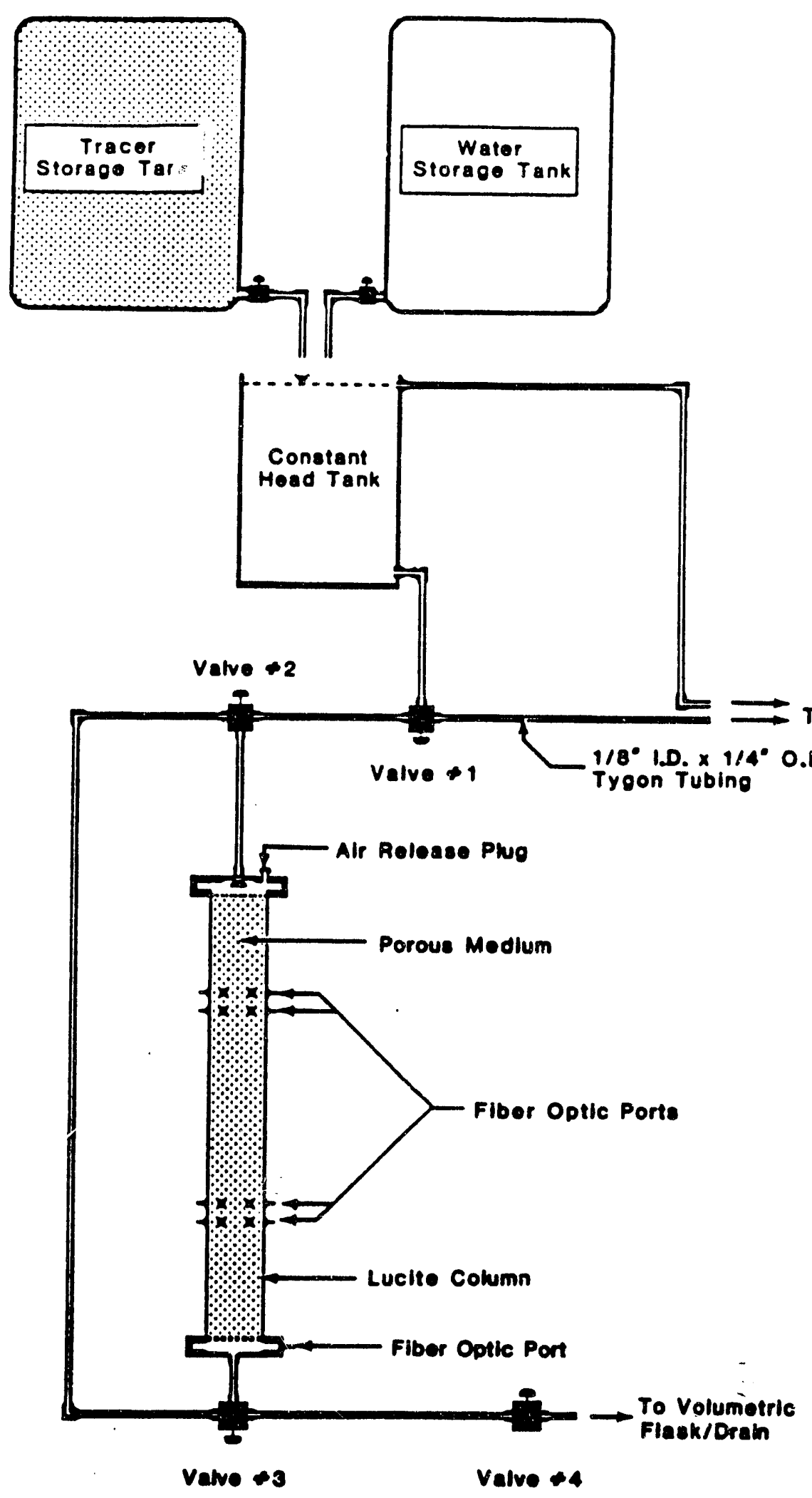

Figure 1. Flow system for the experimental column. 


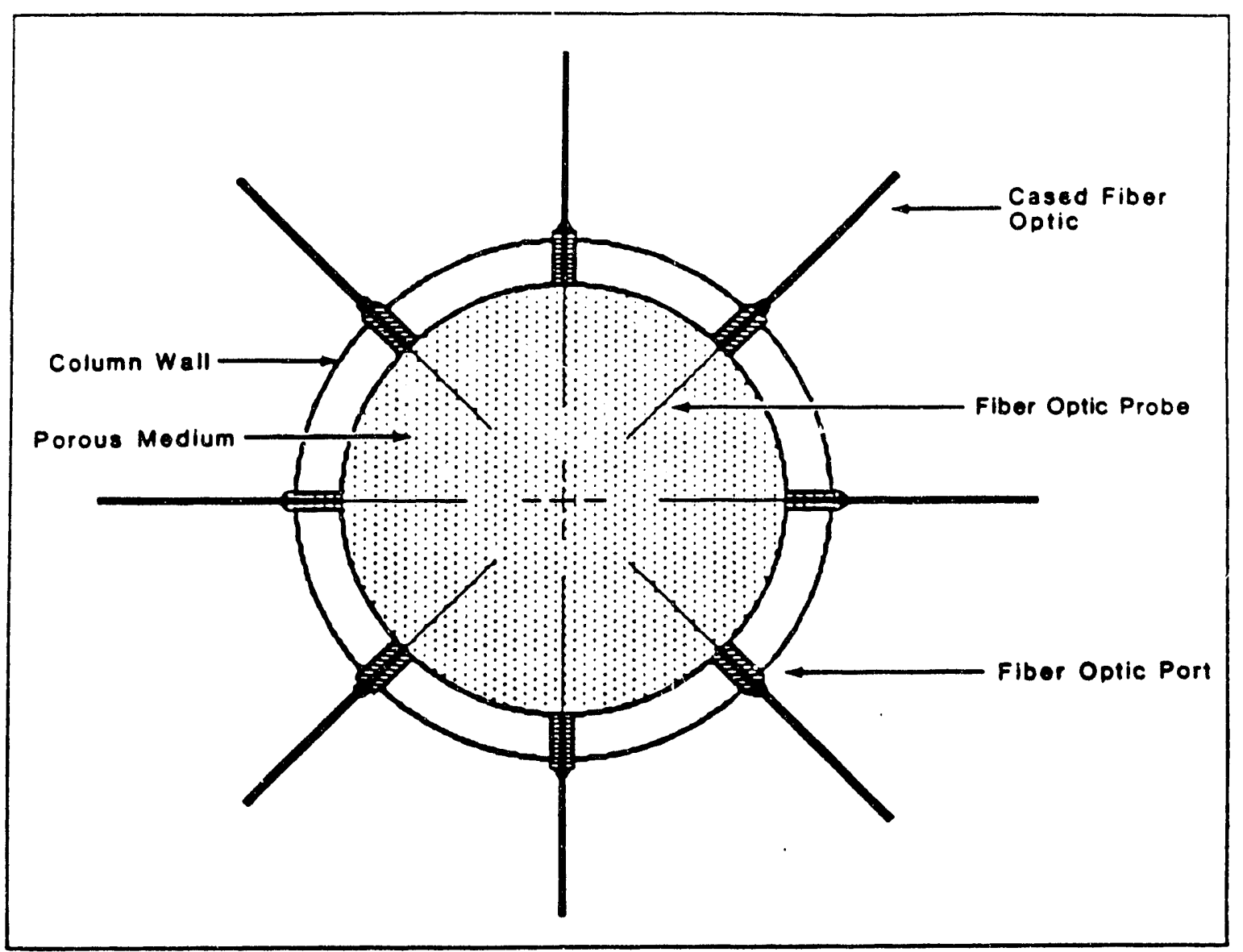

Figure 2. Fiber-optic sensor placement within the column. 

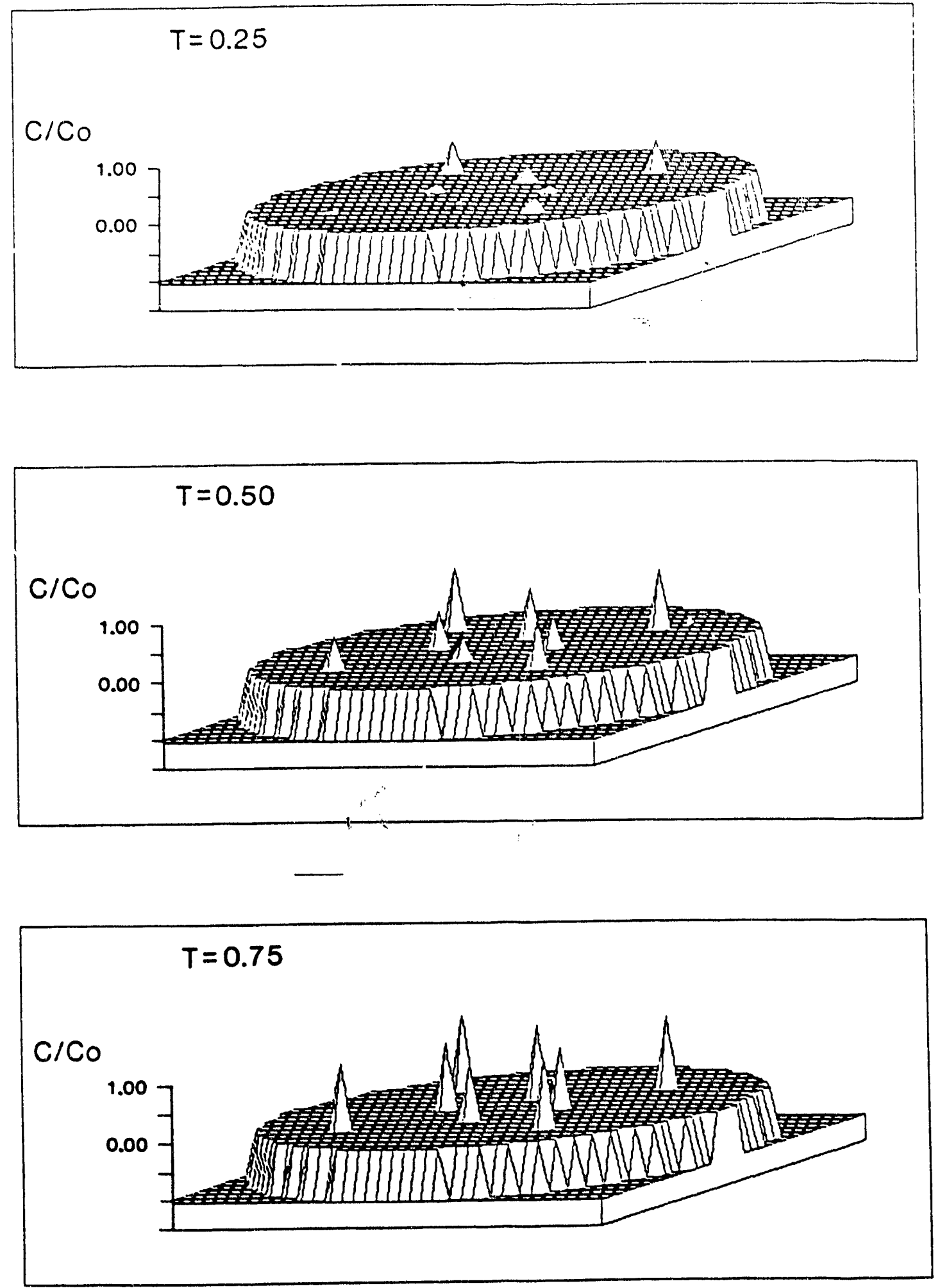

Figure 3. Relative concentration at a distance of $78.1 \mathrm{~cm}$ from the source at three values at relative time $T$. 


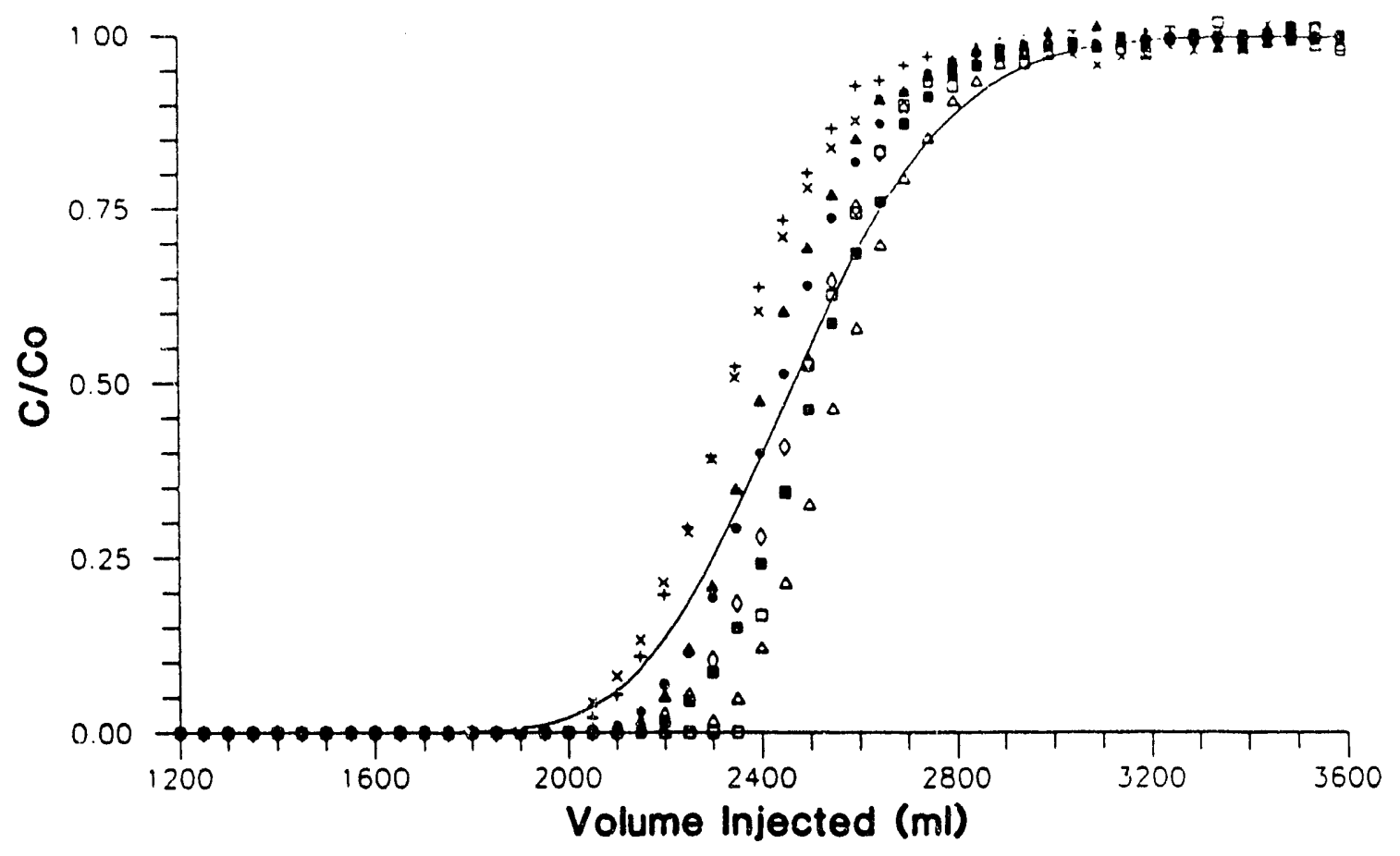

\section{KEY}

Analytical solution
$\Delta \quad$ Fiber 1
- Fibor 2
- Fibar 3
+ Fibor 4
$\triangle \quad$ Fiber 6
$\times \quad$ Flbor 6
- Fiber 7
- FIber 8

Figure 4. Observed (symbols) and theoretical (solid) break through curves. Each symbol corresponds to one observation point. The observation plane is at $X=78.1 \mathrm{~cm}$ and the flow rate is $5 \mathrm{ml} / \mathrm{min}$. 

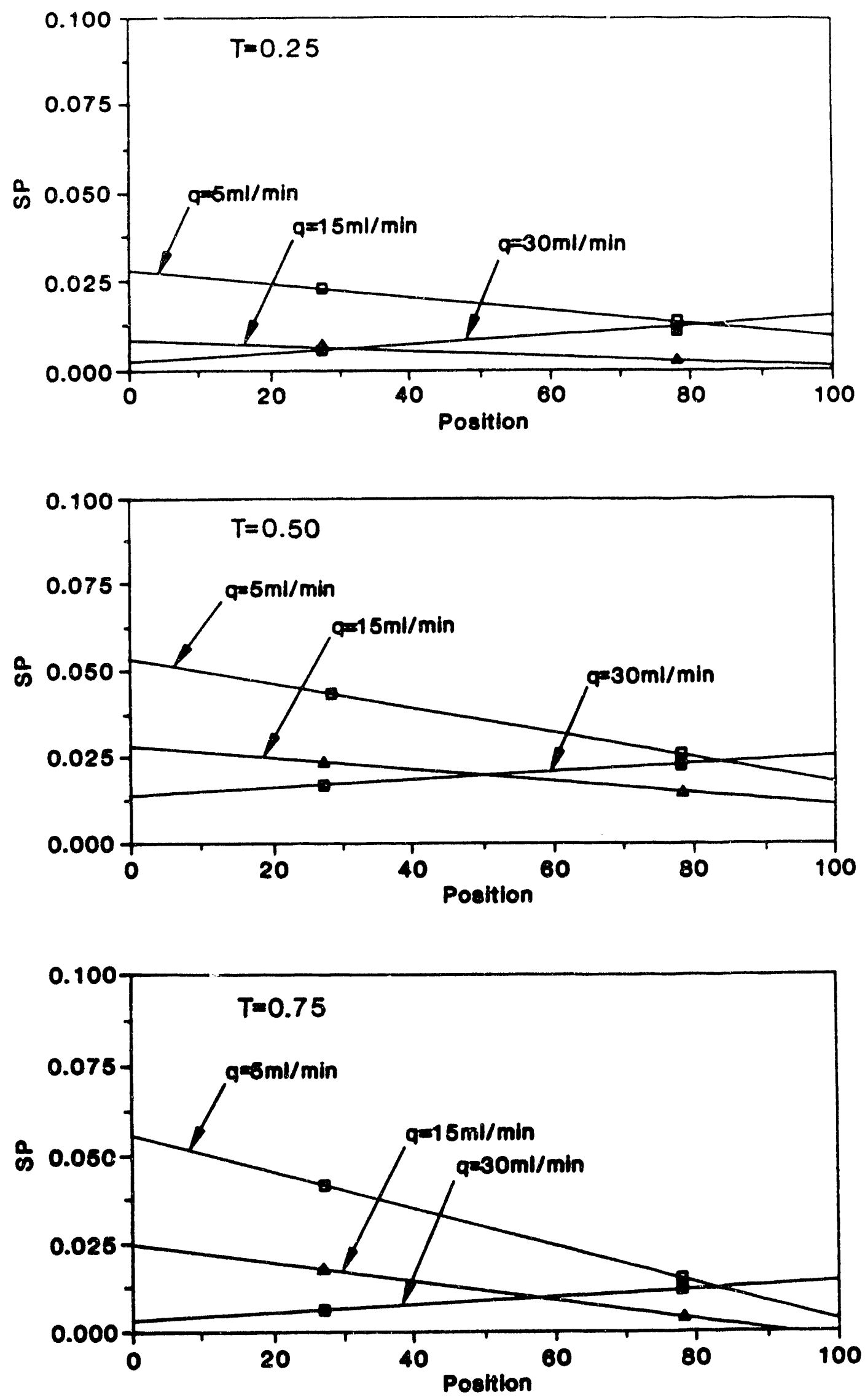

Figure 5. Spatial variability vs. distance from source for three different values of relative time, i.e. $T=0.25,0.50,0.75$, and three flow rates, i.e. $q=5,15,30 \mathrm{ml} / \mathrm{min}$. 

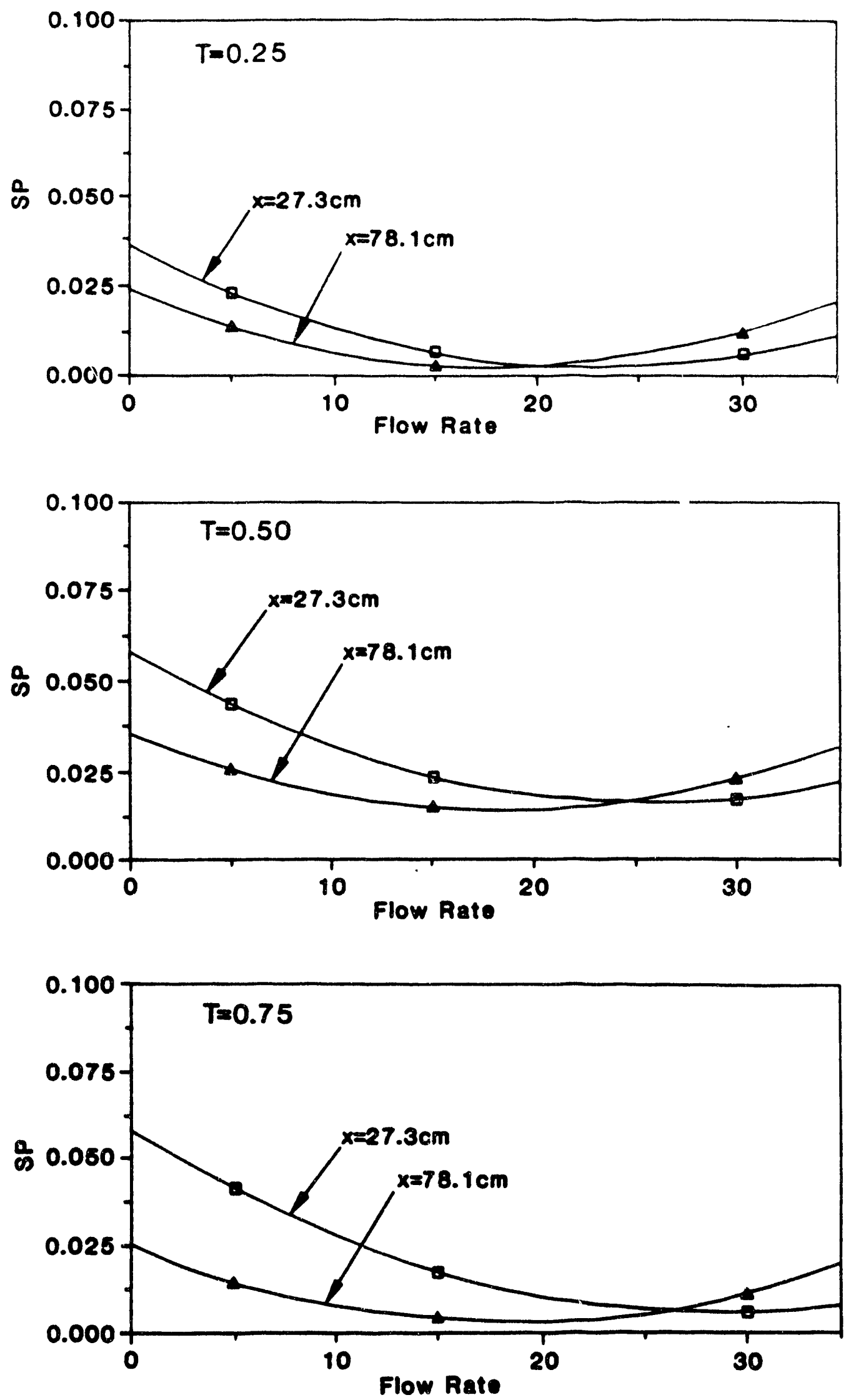

Figure 6. Spatial variability vs. flow rate for two different distances from the source, i.e. $x=27.3$, and $78.1 \mathrm{~cm}$, and three different values of relative time, i.e. $T=0.25,0.50,0.75$. 

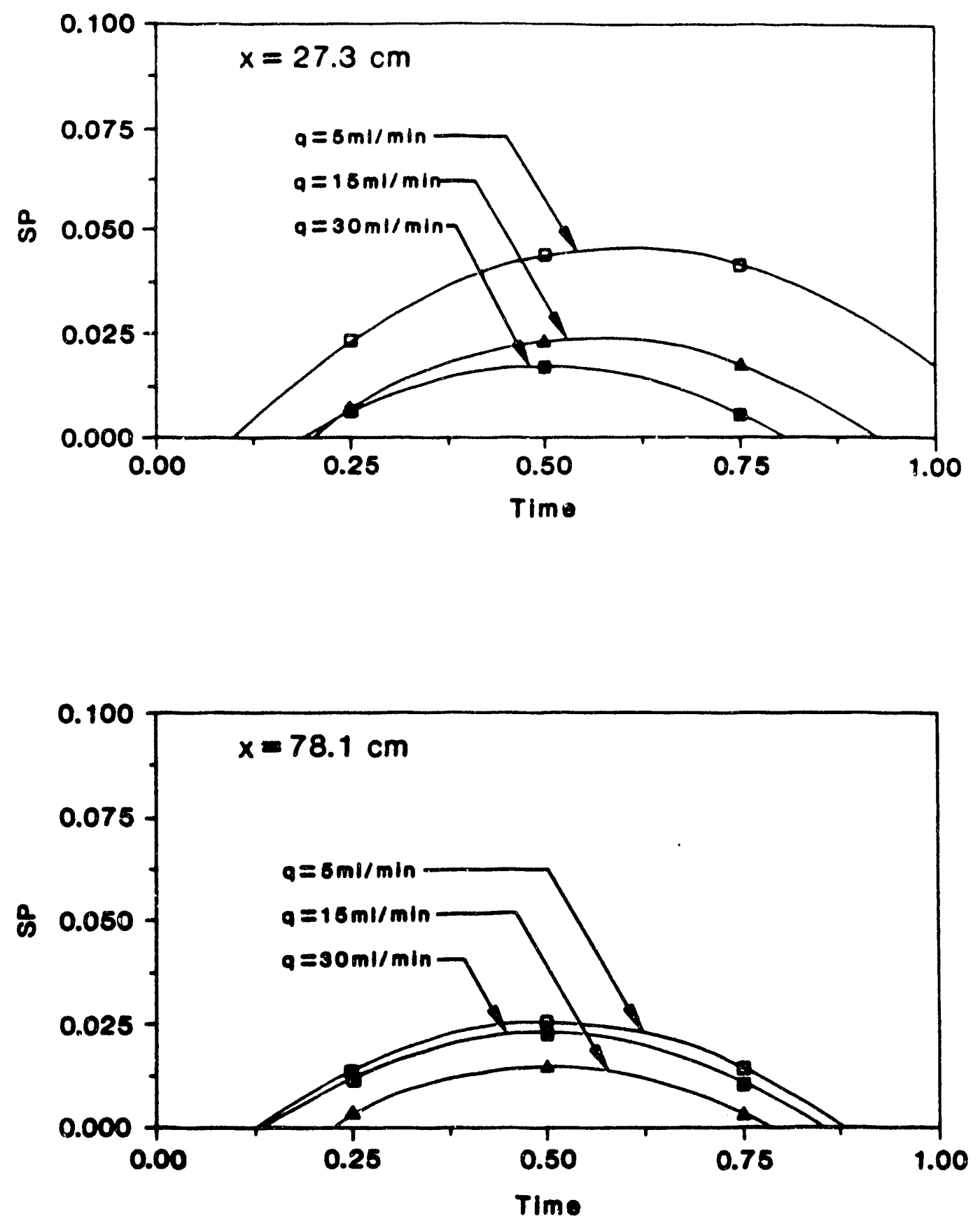

Figure 7. Spatial variability vs. relative time for three different values of flow rate, l.e. $q=5,15,30 \mathrm{ml} / \mathrm{min}$, and two distances from the source, i.e. $x=27.3$ and $78.1 \mathrm{~cm}$. 

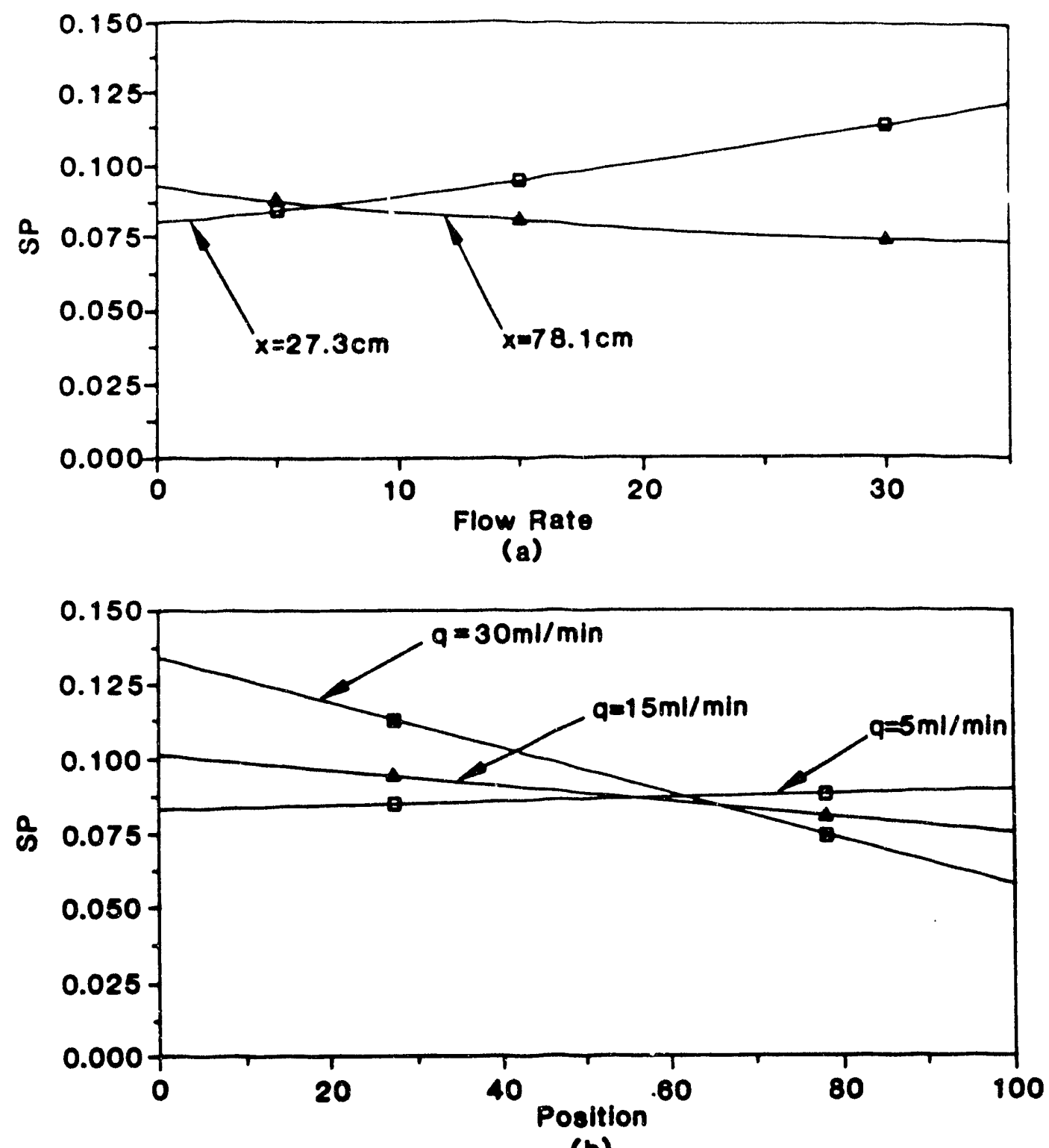

(b)

Figure 8. a) Spatial variability vs. flow rate, i.e. $q=5,15,30 \mathrm{ml} / \mathrm{min}$ for time averaged values of concentration and b) spatial variability vs. distance from the source, i.e. $x=27.3$, and $78.1 \mathrm{~cm}$ for time averaged values of concentration. 

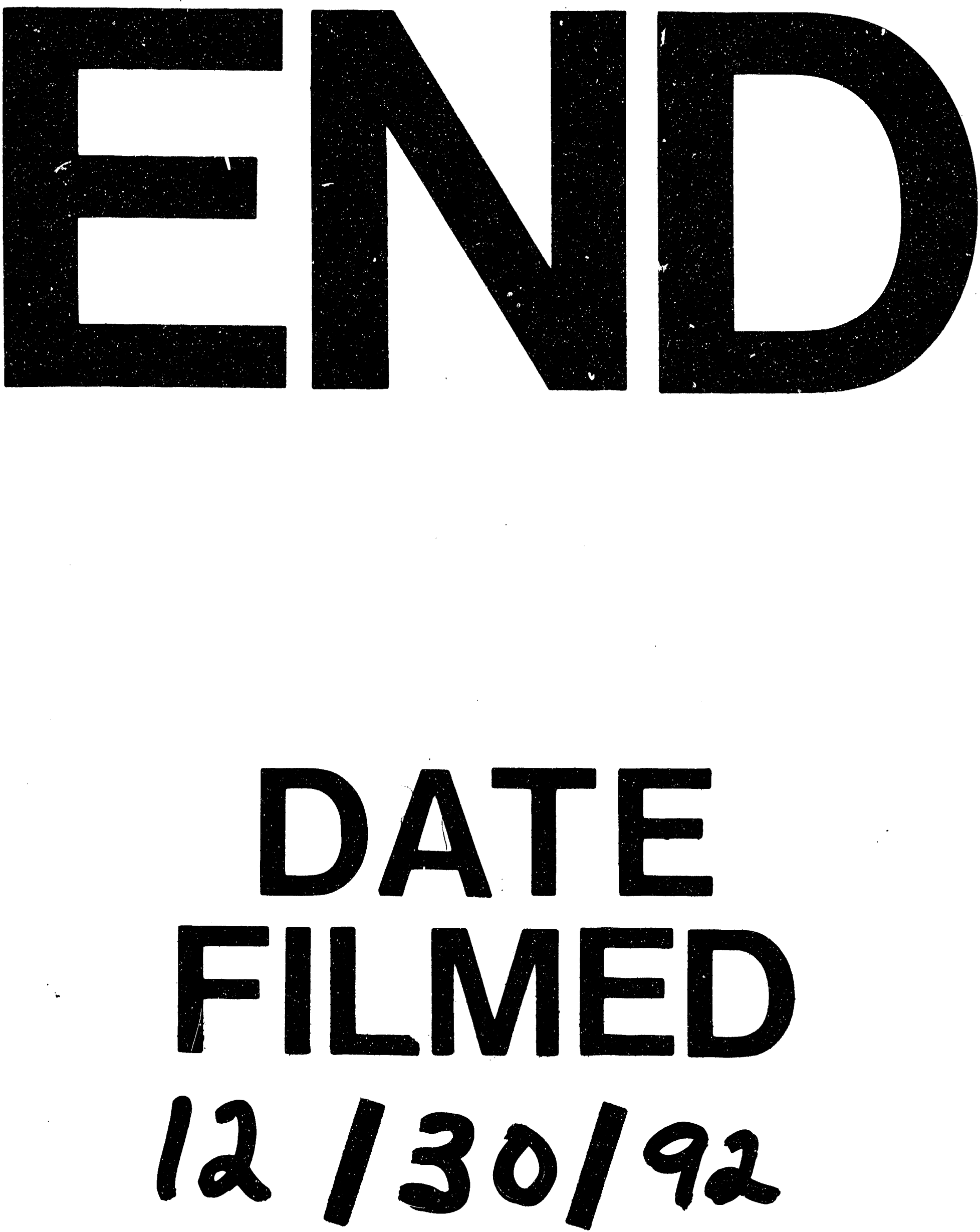
- 